\title{
A windfall for RNA
}

\section{Two 2006 Nobel prizes reflect the central role of RNA in gene regulation and emphasize the interplay of discoveries in chemistry and biology.}

$\mathbf{R}^{\mathbf{s}}$ NA has featured prominently in a series of recent groundbreaking discoveries. In December of 2006, the Nobel committee recognized two such advances by awarding the Nobel Prize in Chemistry to Roger Kornberg for his work on elucidating the molecular mechanisms of RNA transcription and the Nobel Prize in Physiology or Medicine to Craig Mello and Andrew Fire for their discovery of RNA interference (RNAi). In commemoration of these events, this month's issue celebrates RNA and its diverse roles within cells.

For many years, DNA dominated our view of genetics and molecular biology. However, since the elucidation of the genetic code some 40 years ago, genetic and biochemical studies have offered clues that RNA participates more actively in the central dogma than previously thought. The identification of reverse transcriptase enzymes and catalytic RNA presaged the discovery of several previously unknown RNAcentric pathways, including RNA splicing, RNA editing and RNAi. Structural biology methods have also

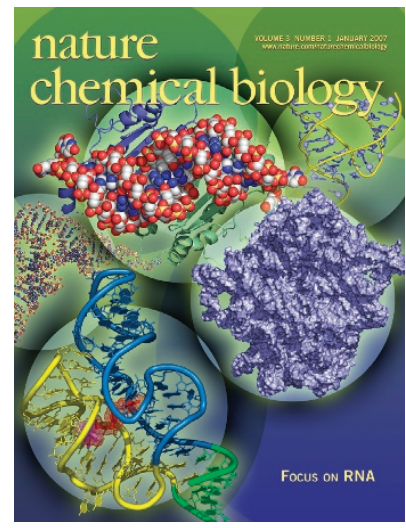

Disputes about what constitutes chemistry present a conundrum for both chemistry and chemical biology. Given the interdependence of these fields, one could even characterize these philosophical differences as counterproductive. Although we do not aim to resolve this question here, it is clear that discoveries at the interface of chemistry and biology have always attracted attention from chemistry Nobel Prize selection committees. For example, Emil Fischer received the second chemistry prize in 1902 for "his work on sugar and purine syntheses" (http:// nobelprize.org). So far, over 30 chemistry prizes have been awarded for chemical biology-related discoveries, which can be grouped into broad classes: isolation and characterization of essential biomolecules, determination of the three-dimensional structures of biological macromolecules and complexes, discovery of new biochemical pathways, and development of enabling technologies for probing biological systems. Put in context, this year's chemistry prize, which involved biochemical and structural analysis of a fundamental aspect of gene revolutionized our understanding of the diversity of RNA molecular structure and function. Structural studies of tRNAs, ribozymes and riboswitches have shown that RNA is capable of folding into threedimensional structures reminiscent of proteins. Landmark structural analyses of the ribosome and RNA polymerase complexes have fundamentally altered our view of translation and transcription, respectively. The rapid pace and surprising outcomes of discoveries in the RNA field makes it apparent that RNA has grown out of its bit part as a simple informational biopolymer and become worthy of consideration as a leading player in cellular biochemistry. Thus it is quite appropriate that the Nobel committee has recognized RNA's central importance with the two prizes this year.

The Nobel prize represents the ultimate recognition of achievement for scientists whose fields were fortunate enough to have been the beneficiaries of Alfred Nobel's legacy. The prizes also have the important practical effects of energizing research fields, inspiring young researchers and providing a convenient way to communicate important scientific achievements to a broader audience. Despite the great excitement that accompanies the announcement of the Nobel Prizes, disagreements sometimes arise about the selection of awardees within a research area or the choice of which scientific discoveries to honor. This year was no exception. For example, some plant biologists felt that seminal discoveries supporting RNAi pathways in plants should have been included in the Physiology or Medicine prize (for example, see Nature 443, 906, 2006). In addition, certain chemists disagreed with the Nobel committee's decision to award the chemistry prize for transcription, which some consider a fundamentally biological discovery (Nature 443, 615, 2006). regulation, offers an excellent example of chemical principles and methods being applied to understand biological systems.

Though it is true that recent years have witnessed an increase in the number of chemistry prizes awarded for 'more biological' discoveries, fundamental chemistry will continue to garner recognition by the Nobel committee. Perhaps the recent awards reflect that many of the current 'big questions' in chemistry are being posed at the interface of chemistry and other disciplines such as biology and physics (Nature 442, 500-502, 2006). In our view, there could be no greater testament to the importance and utility of chemistry than the fact that it has become fundamental to our modern understanding of biology.

To celebrate the achievements of the RNA community, this issue features a collection of articles that highlight current themes at the frontier of RNA chemical biology. Our authors examine the central dogma of molecular biology, focusing on the application of chemical ideas and approaches for understanding transcription, RNA processing and the genetic code. Several articles delve into the mechanistic basis and therapeutic potential of RNAi and underscore the importance of structural biology for offering new insights into RNA function. New studies on the lysine riboswitch suggest that these RNA regulatory elements may offer valuable therapeutic targets, and a Perspective examines the application of natural and engineered RNA components for building synthetic genetic circuits. Finally, we discuss how interdisciplinary collaboration has been essential to the rapid pace of discovery. These articles provide a sampling of the exciting advances being made in the RNA field, which we fully expect will be expanded by future research on RNA structure and function by chemical biologists. 